sound effects on very long waves. For this purpose he took to the Antarctic special apparatus for recording whistlers on a tape recorder, made in the Geophysical Institute of tho Czechoslovak Academy of Sciences. He performed measurements during the journey to

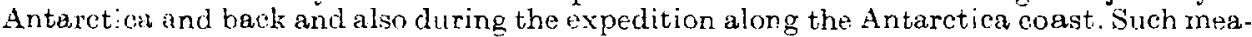
surements were carried out for the first time ever within the framework of Soviet Antarctic expeditions.

The material obtained during the expedition is rich and interesting. It will now have to be elaborated in detail. subjected to a thorough analysis, compared with the material of Czechoslovak stations and the appropriate scientific conclusions reached.

Jaroslav Subrt

\title{
STÄNDIGE POLNISCH-TSCHECHOSLOWAKISCHE ZUSAMMENARBEIT BEI DER METEOROLOGISCHEN UND KLIMATISCHEN ERFORSCHUNG DER. TATRA
}

Vom 14.- 19. XI. 1960 wurde in Bratislava in den Räumlichkeiten der Slowakischen Akad. d. Wiss. eine Konferenz der polnisch - tschechoslowakischen ständigen Komission fïr die meteorologische und klinatische Erforschung der Tatra abgehalten. Es war dies die 6. Zusarnmenkunft dieser Komission, deren Aufgabe es ist, die meteorologische Erforschung des Gebiets der Tatra in beiden Stałtsterritorien zu leiten, wobei die Eriorschung durch Messungen im 'Terxain verwirklicht wird. Im Sommer wird auf der Linie Liptovsk: Hrádok - Zakopane die Veränderung der meteorologischen Elemente in Abhängigkeit von Exposition und Meereshöhe geprift, im Winter wird die Verteilung der Schneedecke, ihr Wasserwert und die übrigen physikalischen Eigenschaften der Schneedecke untersucht. In gomeinsamer Arbeit wurłen aû diese Weise seit 1958 zwei Terrainmessungen im Sommer und 2 im Winter durchgeführt, worüber besondere Abhandlungen veröfentlicht werden sollen. Femer bereitet die hommission eine Monographie des Klimas der Tatra vor. Sie behandelte bereits die methodischen Fragen der Vergleichung des Materials, da die beiden staatlichen Dienste weder ein\}eitliche Beobachtungsvorschriften noch gleiche Beobuchtungstermine haben. Auch der Inhalt der Monographie wurde schon besehlossen: gerade in der letzten Konferenz in Bratislava wurde die struktur der Monographie vercinbart und die Autoren der einzelnen Abhandlungen wurden bestimmt. Man kam überein, daß die Monographie über das Klima der Tatra folgende Kapitel aufweisen soll: Fiach dem Vorwort und der Einleitung werden die physisch-geographischen Verhältnisse des Gebietes bewertet, ferner werden die Sonnenstrahlung, die Luit- und Erdbodentemperatur, ov. die Temperatur des Wassers in den Seen, der Luftchruck, der Wind und dio Verdunstung, die Bewölkung und der Sonnenschein, die vertikalen und horizontalen Niederschläge, die Schneedecke, die Lawinen und Gewitter, die Verunreinigung der Luft, die Sicht, das Komplexlslima von 3 auserwählten Orten, die Klimarayonisation des Ge. bietes und die Bewortung des Erdbodenzustandes sowie das Bio- und Mikroklima behandelt. Fine Zusammenfassung schließt den Band ab. Der Umfang der Monographie wird auf ungefähr 700 Seiten geschätzt, 'Tabellen und Abbildungen nit eingerechnet, außerdem werden ca 120 klimatische Karten des Gebietes die Monographie varvollständigen. Das Werk soll in polnischer und slowakischer Sprache veröfentlicht werden, wobei ausfïhrliche fremdsprachige Zusammenfassungen in den Weltsprachen beigefügt werden. Das Manuskript der Monographie soll im J. 1963 beendet sein. Es soll die Auswortung der klimatischen Elemente des Zeitabschnittes 1931 - 1960 als Unterlage benützen. Wobei freilich bei Stationen mit längeren Beobachtungsreihen auch dieses Material umfaßt werden soll. Dies betrifft die Bewortung der säkulären Veränderungen im Klima der Tatra.

Die Beratungen der ständigen Kommission fanden absechselnd in Zakopane and Stary Smokovec, resp. in Bratislava statt. Die Komission setzte sich zusammen aus Mitarbeitem der Polnischen meteorologischen und hydrologischen Gesellschaft und des polnischen Staatsinstitutes für Hydrologie und Meteornlogie (PIHM), Vertretern der mateorologischen Abteilungen der 'Tschechoslowakischen Akad. d. Wiss., der Slowakischen Akad. d. Wiss. und der Tschechoslowakischen Akad. der landwirtschaftlichen Wiss. ferner ans Mitarbeitern des Laboratoriums für Meteorologie und Klimatologie bei der Komensḱ́ -Universität in Bratislava, sowie aus Mitarbeitern des Hydrometeorologischen Institutes in Prag und Bratislava. Die Beratungen führte von polnischer Seite meistens Doz. M. Molga und von tschechoslowækischer Seite Prof. M. Konček.

St. Petrovic 